\title{
Dedication: Heinz Floss and Christopher Walsh—pioneers in natural product chemical biology
}

\author{
Rolf Müller ${ }^{1,2} \cdot$ Gerard D. Wright ${ }^{3,4}$
}

Published online: 7 February 2019

(c) Society for Industrial Microbiology and Biotechnology 2019

\section{Introduction}

In January 2018 the Society for Industrial Microbiology and Biotechnology (SIMB), the Korean Society for Microbiology and Biotechnology (KMB), and the Society for Actinomycetes Japan (SAJ) cosponsored the Natural Products Discovery and Development in the Genomic Era. This meeting, attended by researchers from across the globe, was dedicated to two pioneers in the field of natural products-Heinz Floss and Christopher Walsh. These investigators helped lay the foundation for natural product research as it progresses in the Genomic Era. Combined, they contributed to our understanding of the details of the biosynthesis of a significant number of antibiotics, anticancer agents, and many other microbial natural products.

The fruits of the large body of work contributed by Floss and Walsh can be seen in their combination over 1300 publications that have served to define mechanistic natural product biosynthetic chemical biology over the past $4 \mathrm{dec}-$ ades, the number of successful companies, large and small, they have advised, and perhaps most importantly, the quantity and quality of researchers that have trained under their

Gerard D. Wright

wrightge@mcmaster.ca

Rolf Müller

Rolf.Mueller@helmholtz-hips.de

1 Helmholtz Centre for Infection Research (HZI), Helmholtz Institute for Pharmaceutical Research Saarland (HIPS), Saarland University Campus Building E8.1, 66123 Saarbrücken, Germany

2 Department of Pharmacy, Saarland University Campus Building E8.1, 66123 Saarbrücken, Germany

3 Michael G. DeGroote Institute for Infectious Disease Research, McMaster University, Hamilton, ON, Canada

4 Department of Biochemistry and Biomedical Sciences, McMaster University, Hamilton, ON, Canada supervision who are their tangible legacy and continue to advance the field of natural product research.

At the meeting, the outcomes of this foundational influence were in evidence. Currently, the field of natural products is experiencing a renaissance, neatly paralleling the historical renaissance of the 15th and 16th centuries that saw the rediscovery of classical art and science following a period of intellectual regression and stagnation rightly known as the Dark Ages. The advances of natural product chemistry and biology were profoundly felt in the mid-20th century with the development of analytical techniques for their purification and characterization along with an understanding of the basic principles of their biosynthesis using technologies such as stable isotope incorporation along with chemical and biochemical studies. However, it is the application of natural products on the development of drugs for humans and animals ranging from antibiotics and anticancer agents, to insecticides, nematicides, and immune suppressors that distinguishes this field from many others. Floss and Walsh contributed significantly to this era, but it is their work in the 'natural product Dark Ages'-the 1980s to early 2000s-that has been revolutionary. These Dark Ages are defined by a pivot in industry and academia away from natural products in drug discovery toward high-throughput chemistry and screening of smaller, less complex chemical entities. This marginalized the impact of natural products on the development of new antibiotics and other drugs.

At the same time, Floss, Walsh, and a select few others toiled to advance natural product research through the multidisciplinary application of new technologies in molecular biology and biochemistry, focused through the lens of rigorous chemical thinking. The results are nothing short of transformative, providing fundamental understanding of the logic of, for example, assembly-line biosynthesis of polyketides such as the ansamycins, and non-ribosomal peptides such as vancomycin. Their insights into linking the discovery of natural product biosynthetic gene clusters and the transformative chemistry required for the assembly of a final product 
that is chemically complex and bioactive are too numerous to mention but are grounded in their personal scientific development and their leadership in mechanistic chemical biology. These contributions launched the Genomic Era of natural product research that we are currently experiencing.

\section{Heinz Floss}

Heinz Floss was born and raised in Berlin, Germany, where he finished high school in 1953. After initial basic courses of studies in chemistry, he chose to study with Friedrich Weygand for his "Diplomarbeit" (master's thesis) [8]. Weygand was a student of Richard Kuhn (Nobel Prize 1938), himself a student of Richard Willstätter (Nobel Prize 1915) who worked with Adolf v. Baeyer (Nobel Prize 1905), a student of August v. Kekulé whom himself worked with Justus von Liebig. Floss was supervised by one of Weygand's assistants, Helmut Simon when Weygand accepted an offer from Technical University of Munich which made all of them migrate to Bavaria. For his Ph.D., Floss chose to join a collaboration with Kurt Mothes in Halle on the biosynthesis of ergot alkaloids for which he made radiolabeled versions of mevalonic acid which had just been identified as the precursor of isoprene units. Corresponding feeding experiments established the building blocks of the ergoline ring system as tryptophan, the methyl group of methionine and an isoprene unit derived from mevalonic acid, setting a new biosynthetic paradigm as the accepted doctrine at that time postulated that alkaloids and terpenoids do not co-occur in nature. Floss made biosynthesis of ergot alkaloids a lifelong project with almost 100 resulting publications. He managed to overcome numerous hurdles typical for collaborations with former East German academics across the iron curtain but also developed a close friendship with Detlef Gröger, assistant with Kurt Mothes and later Professor at the Academy of Sciences Institute in Halle.

From Munich, Floss moved to become a postdoctoral researcher with Eric E. Conn at the University of California, Davis. During that stay, he visited Varro E. (Tip) Tyler, who as ergot researcher had spent a sabbatical year at Mothes' institute in Halle. Tyler had just accepted to become Dean of the Pharmacy School at Purdue University and offered Floss a faculty position in the Department of Medicinal Chemistry which Floss accepted, sight unseen. The Floss laboratory widened the scope of its activities at Purdue to become involved in the stereochemistry and mechanistic details of enzyme reactions. Specifically, they tackled and biochemically characterized enzymes of the shikimate pathway, pyridoxal phosphate-dependent enzymes and biocatalysts installing chiral methyl groups.

The second main area Floss started at Purdue was the biosynthesis of different antibiotics, initially using feeding experiments employing stable isotope-labeled precursors and later more and more moving into enzymology and deciphering the genetic basis of secondary metabolite formation. Most of these compounds were discovered by Tübingen microbiologist Hans Zähner who closely worked with the Floss lab for many years. In the course of the antibiotics work, Floss engaged in a collaboration with David Hopwood at the John Innes Institute in Norwich and Satoshi Ōmura (Nobel Prize 2015) at the Kitasato Institute in Tokyo to form a team who demonstrated for the first time the feasibility of genetically engineering the biosynthesis of hybrid antibiotics [4]. The authors showed that by integrating some of the biosynthesis genes of actinorhodin into the medermycin producer a new compound, named mederrhodin, could be biosynthesized. One can state that this is the first report ever in which transplanting genes led to the formation of novel chemistry and thus set the stage for all sorts of biosynthetic engineering and synthetic biology approaches that are nowadays practiced in the field of combinatorial biosynthesis.

It was clearly the early marriage of chemistry and genetics which led the whole field into an area of enormous excitement, especially those laboratories which managed to embrace topnotch technologies of both fields with leading impact from the Floss and Walsh laboratories. The Floss group extended their efforts in a period of collaboration with William R. Strohl following the laboratories move to The Ohio State University in 1982 and ended up becoming a full-fledged molecular biology group once Floss had made his final move to the Department of Chemistry at the University of Washington in 1987. Here, the primary goal of research became the ansamycin antibiotics containing the so-called $\mathrm{mC}_{7} \mathrm{~N}$ unit AHBA (3-amino5-hydroxybenzoic acid). In establishing and characterizing a new variant of the shikimate pathway introducing the amino group in a DAHP synthase type of reaction, the Floss lab set the stage to clone and sequence the gene clusters for the formation of rifamycin B and ansamitocin P-3. In collaboration with Richard Hutchinson from Wisconsin, the giant type I polyketide synthase was sequenced together with all downstream genes required for rifamycin biosynthesis [1]. At the time of Sanger sequencing, this represented a heroic effort which was published in parallel with the Schupp group at Novartis who had also studied the formation of this clinically important RNA polymerase inhibitor. It is worth mentioning that this was only the second example of a complete bacterial type I polyketide synthase to be published after the seminal work on erythromycin by Peter Leadlay and Leonhard Katz. The Floss group continued their efforts to decipher formation of the AHBA unit biochemically, a highly complex task that was solved with the help of John Frost at Michigan State University who demonstrated that the missing link, the imine of erythrose-4-phosphate, serves as a substrate for the aminoDAHP reaction in a complex pathway starting from kanosamine. Another key finding of the rifamycin work was that 
the pathway instead of a typical lactone-forming thioesterase encodes an amide synthase that was shown to be in charge of the lactamization of the nascent polyketide. When the encoding gene was inactivated, it was observed that instead of just releasing the expected undecaketide intermediates the mutant produces a whole series of linear molecules representing each and every postulated biosynthetic intermediate from the tetraketide to the undecaketide. This result, again published in parallel with the Novartis group, provided the first direct chemical evidence for type I PKS indeed operating in a processive fashion [10]. It also demonstrated for the first time that the enzymatic complex processes multiple nascent chains simultaneously and that the benzenoid to naphthalene ring conversion occurs on the PKS. Delving further into ansamycin work, the Floss group engaged in another fruitful collaboration with Eckhard Leistner from Bonn University. Floss and Leisatner had also studied the formation of ergot alkaloids in plants and fungi. Intriguingly, although the ansamitocins do not look like plant secondary metabolites, they belong to the maytansinoids which are incredibly potent antitumor agents from higher plants. Surprisingly, ansamitocins were soon also isolated from Actinomycetes and were shown to be encoded in a biosynthetic gene cluster forming two subclusters, a first demonstration of biosynthetic genes not necessarily located in a single genomic region [9]. Another peculiarity of ansamictocins is the use of a previously unknown polyketide extender unit, 3-methoxymalonyl-ACP or the "glycolate" unit which was later shown to be used widely in secondary metabolite biosynthesis.

Besides being a world-class scientist and superb mentor for numerous next-generation researchers, Heinz Floss enjoyed traveling, being with friends and became an admirer of oriental art. For quite a while he put more than $5000 \mathrm{~km}$ between his Summer and Winter homes in Seattle and Florida. He graduated $70 \mathrm{Ph} . \mathrm{D}$. students and mentored roughly 75 postdocs plus many visiting professors. One of his early postdoc aficionados, John Vederas, Canada Research Chair in Bioorganic and Medicinal Chemistry in Edmonton, mentioned that "the legacy of a professor is in the education and long-term inspiration of new researchers, who then transmit this in their own way to the next generation. In this regard especially, Heinz has been a true grandmaster who has educated a host of accomplished scientists and profoundly influenced the way a very large field of science has developed." There is little to add to this statement.

\section{Christopher Walsh}

Chris Walsh grew up in Boston and attended Harvard as an undergraduate student, graduating with a degree in Biology and a first author paper in Nature (with Professors John Law and E. O. Wilson) [6] —an auspicious beginning that augured correctly for a stellar academic career. His doctoral work was conducted in the lab Fritz Lipmann (Nobel Prize 1953) under the supervision of Leonard Spector at what is now Rockefeller University. For his postdoctoral work, he chose to study under Robert Abeles at Brandeis University, where he began work on flavoenzymes and mechanismbased inactivators of enzymes, themes that would continue for many years. After a 2-year stint in the Abeles lab, Walsh landed an Assistant Professor position at the Massachusetts Institute of Technology (MIT), jointly appointed in both the Departments of Biology and Chemistry. This was that start of a storied independent career that would span over $4 \mathrm{dec}$ ades, result in more than 800 publications, and involve the training of over 260 graduate students and postdocs.

Walsh's career can be seen as a series of search and destroy missions, to use an imprecise but useful military analogy. He narrows in on a field of great interest, but which has eluded detailed mechanistic study and perhaps is even languishing as a result. He then attacks this target using multiple and often novel techniques, redefining the field, and then moves on. His legacy is one of scholarship and deep insight, bringing chemical thinking to biological problems. He often uses the word 'logic' in his manuscripts and presentations to demonstrate how once unsolvable problems can be dissected by careful experiment and insight into what, in hindsight, seems to make perfect sense.

The 1970s and 80s at MIT saw many of the first missions. Focusing on understanding the chemical biology of enzymatic transformations catalyzed with the involvement of co-enzymes. His work on flavoenzymes, begun in the Abeles lab, ranged across the many redox-associated transformations enabled by this co-factor and launched a foray into other more esoteric redox-active cofactors involved in anaerobic metabolism [7]. Contemporary with this work was a series of inroads into the chemistry of a cofactor that rivaled flavin in versatility, pyridoxal phosphate. The key to success in this work was the application of new technology and strategies to study complex mechanisms. This included mechanism-based enzyme inactivators (suicide substrates) that served to inform on mechanistic pathways and can be often used as drug leads. Additional work from this era included exploration of the mechanisms of C-P bond cleavage in nature, the detoxification of mercury through reduction to the element $\mathrm{Hg}(0)$, which is volatile and diffuses away, and many others. Many of targets of these enzymatic transformations were linked either thematically or directly to natural product chemistry. Walsh also became Chair of the Department of Chemistry at MIT from 1982 to 1987, beginning what would be over a decade of academic administrative leadership.

The 1990s saw a significant refocusing of priorities in the Walsh lab. Coincident with a move from MIT to Harvard Medical School in 1987, where Walsh took up the leadership 
of a new combined department, Biological Chemistry and Molecular Pharmacology, the lab began a series of investigations on a wide variety of medically important enzymes. These included vitamin K-dependent carboxylase, various enzymes required for peptidoglycan biosynthesis such as MurA, MurB, GlmU, trypanothione biosynthetic enzymes important in parasite redox homeostasis, the peptidyl-prolyl isomerases that are the targets of immune-suppressing natural products, various cancer-associated kinases and phosphatases. The latter were the result of increasing interaction with the cancer biology community that emerged no doubt from Walsh's appointment as CEO of the Dana Farber Cancer Institute, a position he maintained while concurrently holding the position of Department Chair at Harvard Medical School.

During this time, the lab fully leveraged the growing facility in gene cloning and protein expression along with atomic resolution structural biology. A signature example is the deciphering of the molecular mechanism of vancomycin resistance that emerged in enterococci in the late 1980s. With Patrice Courvalin of the Institut Pasteur in Paris, Walsh identified the enzymatic mechanisms of three essential enzymes required for resistance and of a two-component regulatory system needed to trigger the production of these enzymes when exposed to the antibiotic. The result is a restructuring of the bacterial peptidoglycan that is accompanied by a 1000-fold decrease in the affinity of vancomycin for the cell wall and clinical resistance [2].

Stepping down from his administrative roles at Harvard Medical School and Dana Farber Cancer Institute in 1995 provided the impetus for yet another 'mission' this time in understanding the various molecular mechanisms of natural product biosynthesis. Again, following a pattern of alighting on a field when new technologies can successfully interrogate it, Walsh was one of the pioneers in using bacterial genomic sequence data to land on natural product biosynthetic clusters. He then systematically applied the tools he used over his career in molecular biology, protein expression and structure determination, careful biochemical measurements, all united by canny instincts for chemical mechanisms. Using this approach, his group informed on the details of assembly line biochemistry of natural product biosynthesis as well as the elaboration of these scaffolds by a plethora of enzymes [3]. Highlights include the complete dissection of the vancomycin biosynthetic pathway, including the enzymes required for the production of nonstandard natural products and carbohydrates, and in similar approaches, the aminocoumarin antibiotics, the protein kinase inhibitor staurosporine, the bleomycin and epothilone anti-cancer agents, and many others. A recurring interest has been heterocycle and macrocycle formation, and Walsh was one of the leaders in determining the mechanisms of ribosomally encoded peptide intramolecular cyclizations in addition to the formation of bridging nitrogen heterocycles in, for example, the biosynthesis of thiopeptides such as thiostrepton [5].

In a class act of 'leaving when you're on top', Walsh decided to close his laboratory at the height of productivity in 2013. His lab family of over 260 trainees all owe him a direct debt of gratitude for his leadership, encouragement and enthusiasm, and inspiration as someone who inspires creativity and risk taking while never straying from scholarship and deep learning. Walsh continues to educate and lead, now through the pen. He regularly authors insightful and timely review articles that link seemingly disparate areas of Chemistry and Biology to reveal heretofore unrecognized chemical logic. He also builds on what is an impressive collection of monographs. He authored three as an active researcher-Enzymatic Reaction Mechanisms (1978), Antibiotics: Actions, Origins, Resistance (2003), Posttranslational Modification of Proteins: Expanding Nature's Inventory (2005), and has published another three, Antibiotics: Challenges, Mechanisms, Opportunities (with Tim Wencewicz, 2016), Natural Product Biosynthesis: Chemical Logic and Enzymatic Machinery (with Yi Tang, 2017), and recently The Chemical Biology of Human Vitamins (with Yi Tang, 2018). In this next phase of his intellectual career, Walsh is exercising what is one of his most significant gifts-the ability to communicate complex ideas in a clear and concise fashion. This skill is reflected in his dedication to education, to the training of new researchers, and through countless seminars and addresses he has given to the cognoscenti and the amateur alike for over 4 decades that have inspired and informed. It is perhaps this contribution that will outlast the 800 scientific publications through the hundreds and thousands that have experienced his mind directly and indirectly.

\section{Conclusion}

The contributions of Floss and Walsh to mechanistic chemical biology of natural product biosynthesis have not only opened up a previously hidden and rich vista of scholarship on natural products, but also revealed biosynthetic schema that can be leveraged in for example synthetic biology approaches to explore existing chemical diversity and even to engineer and deliver new to nature compounds with unexpected bioactivity. As the Enlightenment was the intellectual and cultural inheritor of the Renaissance, we are poised in the Genomic Era to build on the foundational work of pioneers such as Floss and Walsh to take natural products research into new and unexpected arenas. It is through their force of intellect and ability to inspire that we will be able to do so. 


\section{References}

1. August PR, Tang L, Yoon YJ, Ning S, Müller R, Yu TW, Hoffman D, Kim CG, Zhang X, Hutchinson CR, Floss HG (1998) Biosynthesis of the ansamycin antibiotic rifamycin: deductions from the molecular analysis of the rif biosynthetic gene cluster of Amycolatopsis mediterranei S699. Chem Biol 5:69-79

2. Bugg TD, Wright GD, Dutka-Malen S, Arthur M, Courvalin P, Walsh CT (1991) Molecular basis for vancomycin resistance in Enterococcus faecium BM4147: biosynthesis of a depsipeptide peptidoglycan precursor by vancomycin resistance proteins VanH and VanA. Biochemistry 30:10408-10415

3. Fischbach MA, Walsh CT (2006) Assembly-line enzymology for polyketide and nonribosomal peptide antibiotics: logic, machinery, and mechanisms. Chem Rev 106:3468-3496

4. Hopwood DA, Malpartida F, Keiser HM, Ikeda H, Duncan J, Fuji I, Rudd BA, Floss HG, Ōmura S (1985) Production of 'hybrid' antibiotics by genetic engineering. Nature 314:642-644

5. Walsh CT (2018) Nature builds macrocycles and heterocycles into its antimicrobial frameworks: deciphering biosynthetic strategy. ACS Infect Dis 4:1283-1299
6. Walsh CT, Law JH, Wilson EO (1965) Purification of the fire ant trail substance. Nature 207:320-321

7. Walsh CT, Wencewicz TA (2013) Flavoenzymes: versatile catalysts in biosynthetic pathways. Nat Prod Rep 30:175-200

8. Weygand F, Simon H, Floss HG (1961) Über die umlagerung einiger phenyl-dihydroxy-aceton- und phenyl-chlor-hydroxy-aceton-derivate in benzylglyoxal und methyl-phenyl-glyoxal. Chem Berich 94:3135-3144

9. Yu TW, Bai L, Clade D, Hoffmann D, Toezler S, Trinh KQ, Xu J, Moss SJ, Leistner E, Floss HG (2002) The biosynthetic gene cluster of the maytansinoid antitumor agent ansamitocin from Actinosynnema pretiosum. Proc Natl Acad Sci U S A 99:7968-7973

10. Yu TW, Shen Y, Doi-Katayama Y, Tang L, Park C, Moore BS, Hutchison CR, Floss HG (1999) Direct evidence that the rifamycin polyketide synthase assembles polyketide chains processively. Proc Natl Acad Sci U S A 96:9051-9056

Publisher's Note Springer Nature remains neutral with regard to jurisdictional claims in published maps and institutional affiliations. 